\title{
The Physiologic Cipher at Altitude: Telemedicine and Real-Time Monitoring of Climbers on Mount Everest
}

\author{
RICHARD SATAVA, M.D., F.A.C.S., ${ }^{1}$ PETER B. ANGOOD, M.D., F.A.C.S., ${ }^{1}$ \\ BRETT HARNETT, B.S., ${ }^{2}$ CHRISTIAN MACEDONIA, M.D., ${ }^{3}$ \\ and RONALD MERRELL, M.D., F.A.C.S. ${ }^{2}$
}

\begin{abstract}
Advanced wearable biosensors for vital-signs monitoring (physiologic cipher) are available to improve quality of healthcare in hospital, nursing home, and remote environments. The objective of this study was to determine reliability of vital-signs monitoring systems in extreme environments. Three climbers were monitored 24 hours while climbing through Khumbu Icefall. Data were transmitted to Everest Base Camp (elevation 17,800 feet) and retransmitted to Yale University via telemedicine. Main outcome measures (location, heart rate, skin temperature, core body temperature, and activity level) all correlated through timestamped identification. Two of three location devices functioned $100 \%$ of the time, and one device failed after initial acquisition of location $75 \%$ of the time. Vital-signs monitors functioned from $\mathbf{9 5 \%}-\mathbf{1 0 0 \%}$ of the time, with the exception of one climber whose heart-rate monitor functioned $78 \%$ of the time. Due to architecture of automatic polling and data acquisition of biosensors, no climber was ever without a full set of data for more than 25 minutes. Climbers were monitored continuously in real-time from Mount Everest to Yale University for more than 45 minutes. Heart rate varied from 76 to 164 beats per minute, skin temperature varied from 5 to $10^{\circ} \mathrm{C}$, and core body temperature varied only $1-3^{\circ} \mathrm{C}$. No direct correlation was observed among heart rate, activity level, and body temperature, though numerous periods suggested intense and arduous activity. Field testing in the extreme environment of Mount Everest demonstrated an ability to track in real time both vital signs and position of climbers. However, these systems must be more reliable and robust. As technology transitions to commercial products, benefits of remote monitoring will become available for routine healthcare purposes.
\end{abstract}

\section{INTRODUCTION}

$\mathbf{F}$ IVE CLIMBERS DIED during their May 1996 climb of the summit of Mount Everest. This tragedy brought into focus the extraordinary risks all individuals endure in remote and ex- treme environments. The accounts, popularized by Jon Krakauer in Into Thin Air ${ }^{1}$ and Brougton Coburn and David Beshears in Everest, Mountain Without Mercy, ${ }^{2}$ described in detail the hardships and circumstances leading to disaster. Incredibly in that episode, two climbers died

\footnotetext{
${ }^{1}$ Department of Surgery, Yale University School of Medicine, New Haven, Connecticut.

${ }^{2}$ National Aeronautics and Space Administration (NASA), Commercial Space Center for Medical Informatics and Technology Applications (CSC/MITA), Virginia Commonwealth University, Medical College of Virginia, Richmond, Virginia.

${ }^{3}$ Department of Obstetrics and Gynecology, Uniformed Services University of Health Sciences, Bethesda, Maryland.
} 
during a snowstorm within proximity of the safety of the camp. Another was left for dead but actually survived to walk into camp the following morning with frostbite so severe that he lost his nose, right hand, and fingers. Had the expedition members known that two of their colleagues were fallen at a position just outside their tents, or had it been known that one was severely hypothermic but alive with barely detectable vital signs, the outcome may have been more positive. These three tragedies could have been avoided if current technology had been available to the expedition.

A physiologic cipher is a noninvasive identifying tool to measure physiologic status of an individual in real time, through monitoring of vital signs, biochemical, and other parameters with sensors worn by the individual. When concerned with remote locations, such as expeditions or the battlefield, such monitoring also includes geolocation using the Department of Defense global positioning satellite (GPS) system.

The military sector has been developing a number of wearable systems for location and vital-signs monitoring (VSM). ${ }^{3}$ These systems consist of three principal components:

1. The vital sign sensors, including heart rate, temperature, respiratory rate, electrocardiogram (EKG), motion (accelerometers) and pulse oximeter, are currently worn at various sites on the body. They are typically strapped across the chest or wrist or swallowed in pill form.

2. The GPS device is commercially available and accurate to within 0.75 meters longitude and 1.01 meters latitude.

3. The telecommunications system, usually a radio frequency ( $R F$ ) transmission system, is also commercially available but repackaged into a miniaturized wearable configuration.

In remote environments, strategically placed transceivers are required to receive and transmit the signals from the wearable systems to a base station containing the receiver, signal processor, and computer workstation (or laptop computer). Some systems, such as the Sarcos Personnel Status Monitoring or PSM ${ }^{\mathrm{TM}}$ system (Sarcos, Inc., Salt Lake City, UT) also have a hand-held "medic unit" (Fig. 1) that permits a member of the expedition to be in the field and still monitor the vital signs and location of other individuals or members of the squad. During the Mount Everest climbing season in May 1999, an Everest Extreme Expedition was conducted by a team from Yale University School of Medicine in collaboration with the Yale University-NASA Commercial Space Center for Medical Informatics and Technology Applications, Millennium Healthcare Solutions, and The Explorers' Club. The mission had three objectives: (1) to provide advanced medical support to the climbing expeditions from a base camp at 17,800 feet at Mount Everest Base Camp (EBC) from a telemedicine clinic, (2) to test an emerging VSM system for monitoring a physiologic cipher and vital signs of climbers as they ascend toward the summit of Mount Everest, and (3) to assess the cardiovascular adaptation to hypoxia at high altitude. This report concerns the VSM system and the medical implications of a physiologic cipher.

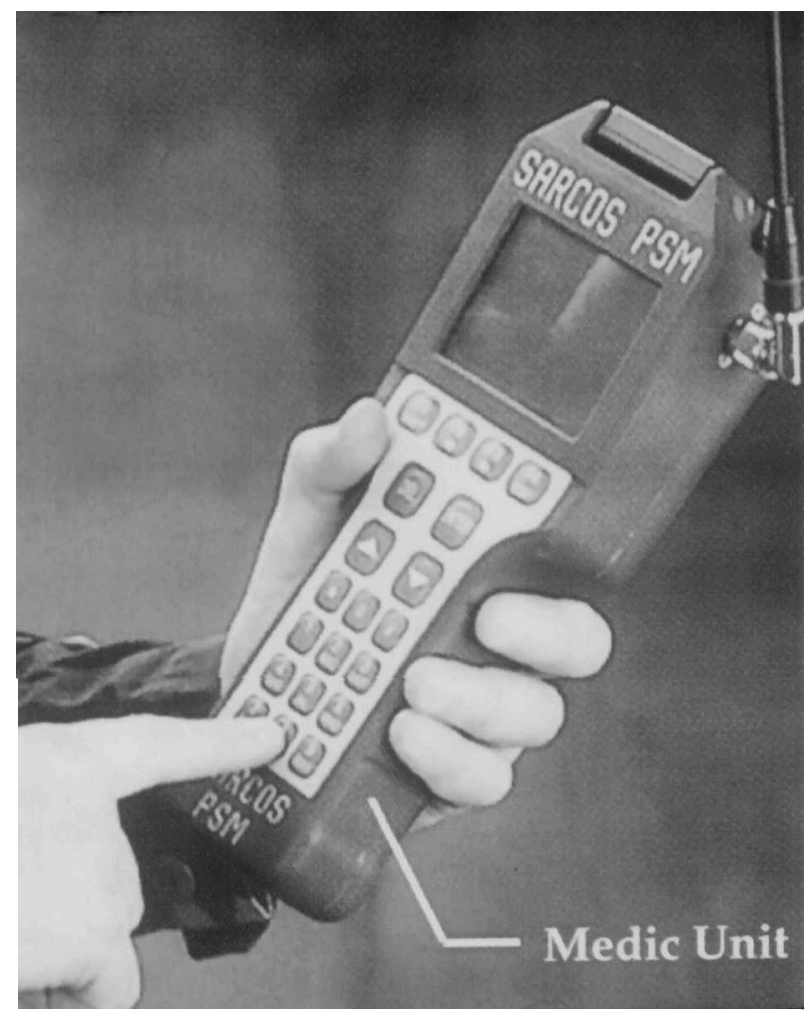

FIG. 1. The medic unit from the Sarcos, Inc. Personnel Status Monitor (PSM) system being developed for the military. (Courtesy of Dr. Stephen Jacobsen, Ph.D., Sarcos, Inc., Salt Lake City, Utah.) 


\section{MATERIALS AND METHODS}

The VSM system was custom designed by FitSense Technologies (FitSense Technologies, Wellesley, MA) in conjunction with requirements by the Department of Defense for the "next generation soldier system." Figure 2 illustrates the three modules which comprise the system:

1. Non-invasive physiologic sensors to measure vital and physical signs. This includes: heart rate (accurate to \pm 4 beats per minute), 3-lead EKG, accelerometer (or chest actigraph, for gross body motion and activity), a surface body temperature monitor (accurate to $\pm 0.01^{\circ} \mathrm{C}$ ), and a core body temperature monitor (ingested pill, accurate to $\pm 0.04^{\circ} \mathrm{C}$ ). The accelerometer is a standard threshold accelerometer with a sensitivity of $0.01 \mathrm{~g}$. The core body temperature pill, similar to the one used by Senator John Glenn during his space shuttle mission, is a silicon-covered jelly-bean size capsule with a temperature-sensitive quartz crystal oscillator in which the signal is inductively coupled to an RF transmitter to an external belt-worn transceiver as described by Mittal et al. ${ }^{4}$ The pill is swallowed and the core body temperature is transmitted every minute to the receiver, which aggregates the data into a data logger.

2. Accurate position tracking using the Global Positioning Satellite (GPS) system (Lassen SK-8, Trimple, Inc., San Jose, CA).

3. Wearable, wireless communication system with radio frequency (RF) transmission using the $928 \mathrm{mHz}$ band. The receiving station at EBC was a laptop computer with a proprietary graphical user interface from FitSense Technologies.

The purpose of the VSM system is to provide a small, unobtrusive monitoring system that can continuously acquire and transmit vital signs and other bio-data in real time while the climbers were performing an ascent. The system is designed for medical research, industrial, and military applications where biodata are recorded while subjects perform normal activities. Currently measured parameters (either directly measured or calculated) include heart rate, core body temperature, surface body temperature (chest and wrist), chest actigraphy (gross body motion), sleep time, and geolocation.

The VSM system was designed to be a modular system which uses an open architecture. The data hub can dynamically receive and store data while reconfiguring as needed to accept

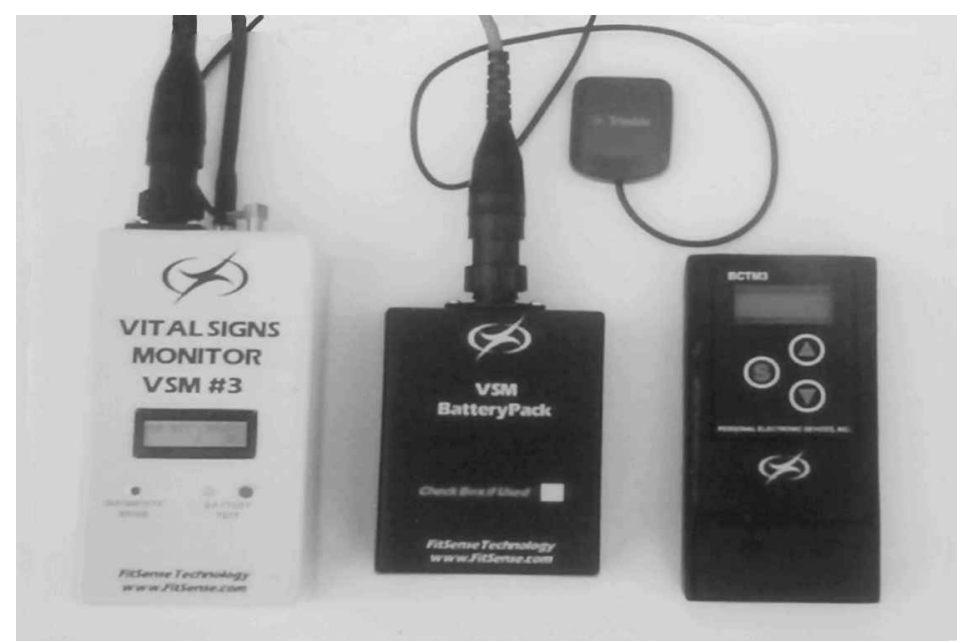

FIG. 2. The vital-signs monitoring (VSM) system of Fitsense, Inc., demonstrating (right to left) the global positioning satellite (GPS) module, the central processing hub, and the radio frequency (RF) transmitter. (Courtesy of Dr. Tom Blackadar, Ph.D., FitSense Technologies, Inc., Wellesley, Massachusetts.) 
new input, with a maximum of 16 sensors per person without RF degradation. The biosensor acquisition is transmitted to the hub on a Personal Local Area Network (PLAN) with digital RF signals. The hub then stores the data or can transmit to a variety of long-haul options, such as CDPD cellular modem or other long haul radio connections.

Each datum is time-stamped and geostamped with the GPS sensor. Time-correlated data from multiple sensors are more robust than an isolated datum, facilitating error correction and datum fusion for superior physiological interpretation. The data may either be stored in the hub or telemetered for remote storage and analysis. For this expedition, the sensor was queried four times per minute until the datum was acquired, then sent to the hub and stored for the subsequent 5-minute transmission. The system hub runs for 10 days on two AA-size batteries storing data once per minute.

The hub comes in three configurations, with integral long-haul radio and GPS, with integral GPS, and with wireless GPS and wireless longhaul radio. The hubs have a direct serial (wired) connection available to the PC for configuration and direct download. The hubs can also communicate through the supersensor. The supersensor can be connected to a Palm Pilot or a personal computer running Windows 95 or Windows 98.

The data from a climber were transmitted over a number of telecommunication links. The initial link was from the climbers using an RF of 918 $\mathrm{MHz}$. Because wireless systems typically require line-of-sight transmission, a device called a "repeater" was positioned on a neighboring mountain (Pomori) to facilitate a vectored path by which the signal could be bounced. This procedure proved quite reliable except when a climber walked into a radio black area, which was occasionally encountered in the fractured glacier topology of the Khumbu Ice Fall. At Base Camp, data were received and compiled onto the laptop. The software was programmed to collect aggregate ASCII (American Standard Code for Information Interchange) datasets from each of the monitoring devices at predetermined intervals. For the expedition, the intervals were every 5 minutes. However, the hub is capable of updating every few seconds.
From the laptop at Base Camp, the data were sent via satellite (Imarsat) using TCP / IP (transmission control protocol/Internet protocol) where they were grounded in Malaysia and routed to an Internet backbone and into a conference room at Yale University School of Medicine in New Haven, Connecticut (Fig. 3). Laplink (Traveling Software, Bothell, WA) was used to download the data from Base Camp to Yale University because of its intuitive interface and reliable transport method. At Base Camp the incremental ASCII datasets were stored in a directory that was indexed to Laplink and marked as "shareable." The physicians at Yale University downloaded the data at 5-minute increments. Because the data sent were standard ASCII, the encoded data were plotted on a graphical user interface, designed by Fitsense Technologies, Inc., which displayed intuitively the ciphered information (Fig. 4). Access to individual continuous display of vital signs (temperature and heart rate) was linked by simply clicking on the climber number, and the chronological graph was plotted (Fig. 5).

In addition to the vital signs, hundreds of images were captured during the expedition using digital photography, digital microscopy, ultrasound, retinal photography, and microcirculation photography. Many of these images were sent to Yale University servers using file transfer protocol. During the daily videoconferences, images were stored in a browser and run in the background for quick reference. Some images, such as ultrasound, were transmitted real-time from EBC.

The technical feasibility of the system was tested during two ascents from EBC to Camp 1 $(19,500$ feet) through the arduous and treacherous Khumbu Ice Fall. This is an exceptionally difficult and dangerous climb, requiring scaling of 40- to 80-foot-high ice cliffs with as many as 8-10 aluminum ladders lashed together (Fig. 6) and traversing over 1,000-foot-deep crevasses on aluminum ladders strapped together, using ropes for a hand railing (Fig. 7). The first trek involved three climbers (two Americans and one Nepalese mountain guide or Sherpa) who were monitored during the trek to Camp 1 and then on the forced return the following day before an oncoming snowstorm. On the second trek, two Sherpas were monitored as they re- 


\section{Real-Time Physiologic Monitoring From Mt. Everest (E3 ${ }^{99}$ )}

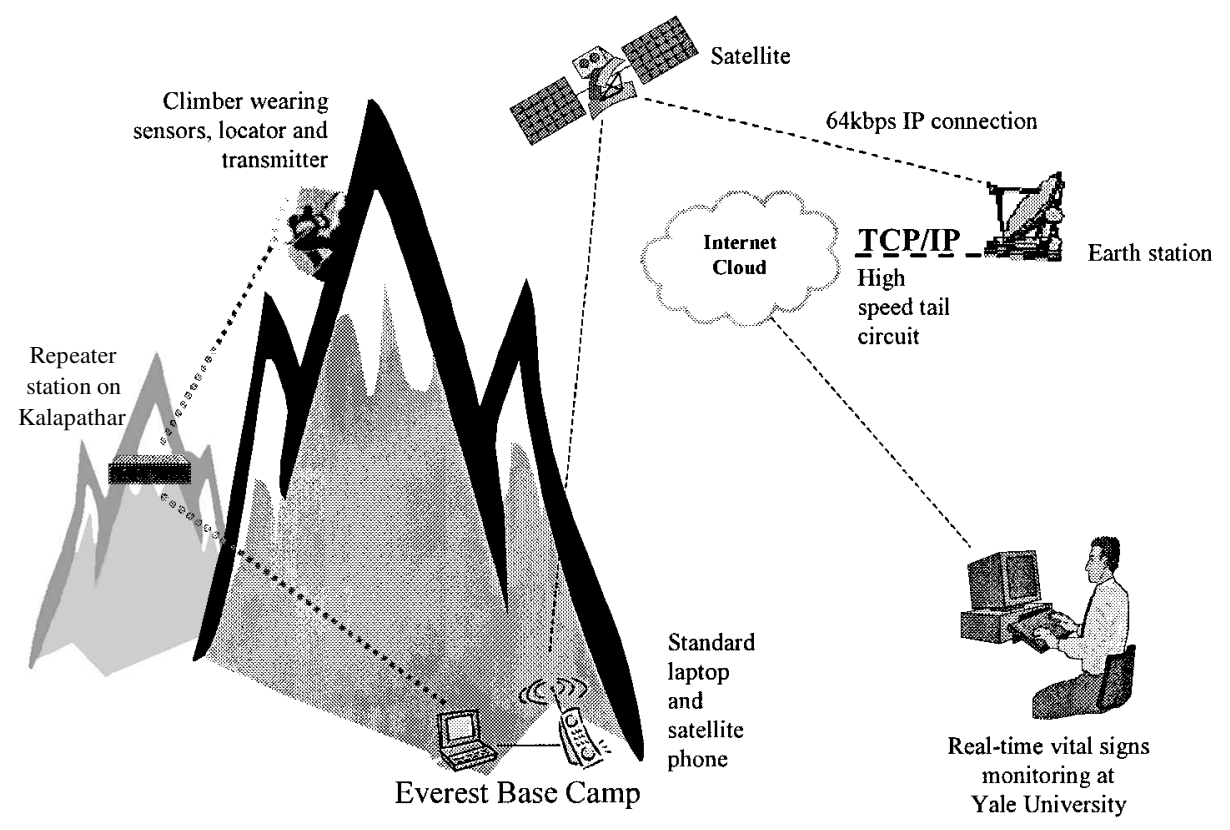

FIG. 3. Diagram of the telecommunications pathway from the individual climber back to the conference room at Yale University School of Medicine (author, BH).

turned to Camp 1 after the snowstorm to retrieve equipment and supplies. The vital signs and position were acquired every 5 minutes and archived and transmitted every 5 minutes. These data (Table 1) consisted of time stamps (using
Greenwich Mean Time), GPS location, heart rate, activity status, skin temperature, and core body temperature. During the daily morning telemedicine conference between Yale University and EBC on the day of the trek to Camp 1, vital signs

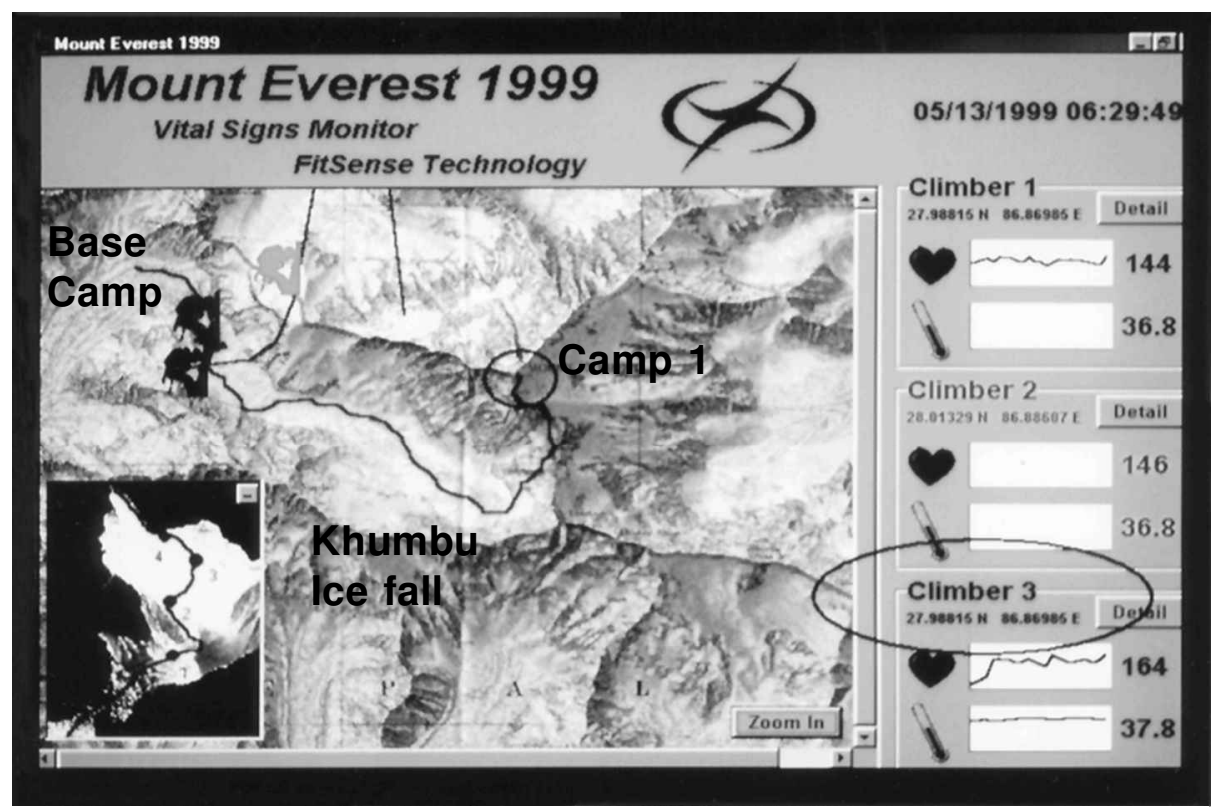

FIG. 4. The laptop computer screen, showing the intuitive graphical interface. On the left is the terrain map of Mount Everest with the overlay of the climbers' position, and on the right are the individual climber's vital signs. (Courtesy of Dr. Tom Blackadar, Ph.D., FitSense Technologies, Inc., Wellesley, Massachusetts.) 


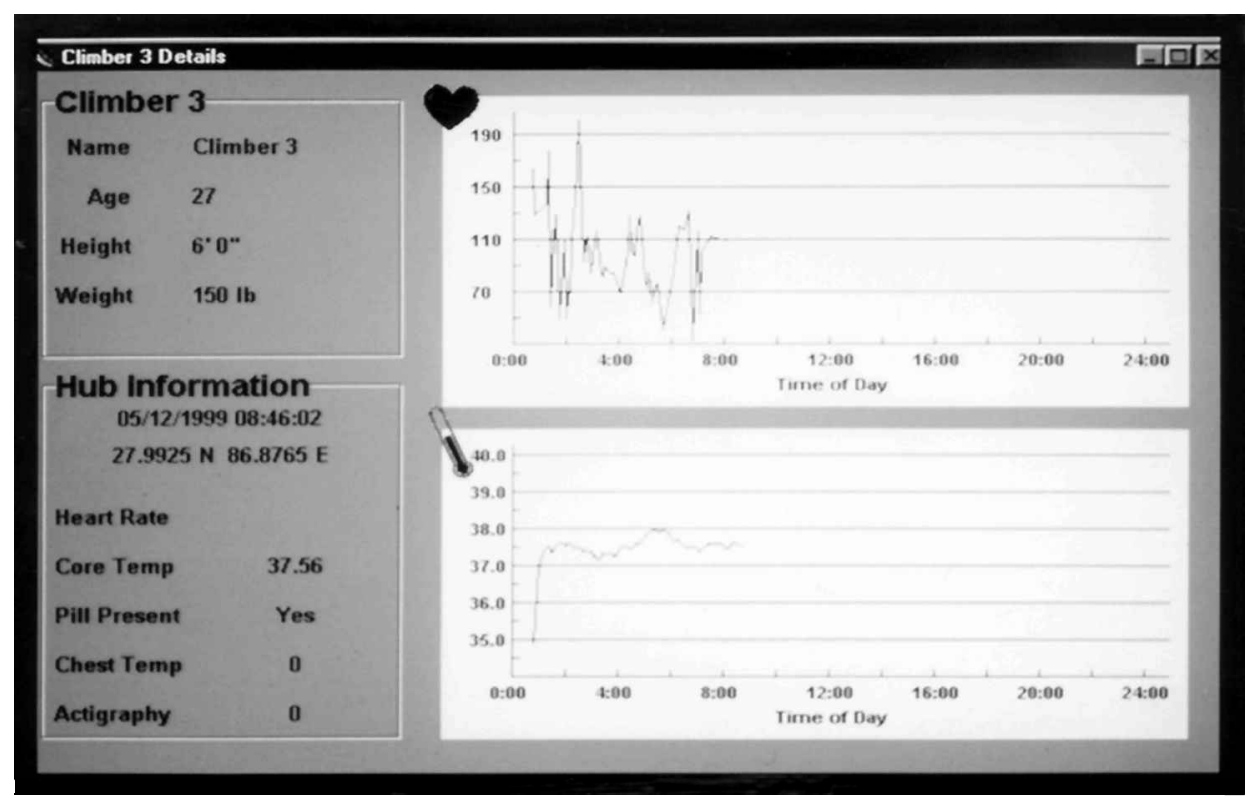

FIG. 5. Chronological graph of the vital signs of Climber 3. (Courtesy of Dr. Tom Blackadar, Ph.D., FitSense Technologies, Inc., Wellesley, Massachusetts.)

were retransmitted to Yale University in real time from the climbers, allowing physicians at Yale University to follow vital signs and location while the climbers were ascending through the icefall. Vital signs were updated every 5 minutes back at Yale University.
During the climb into the Khumbu Icefall, more than 4,000 data points were logged for analysis.

Institutional Review Board approval was obtained through the Yale University School of Medicine.

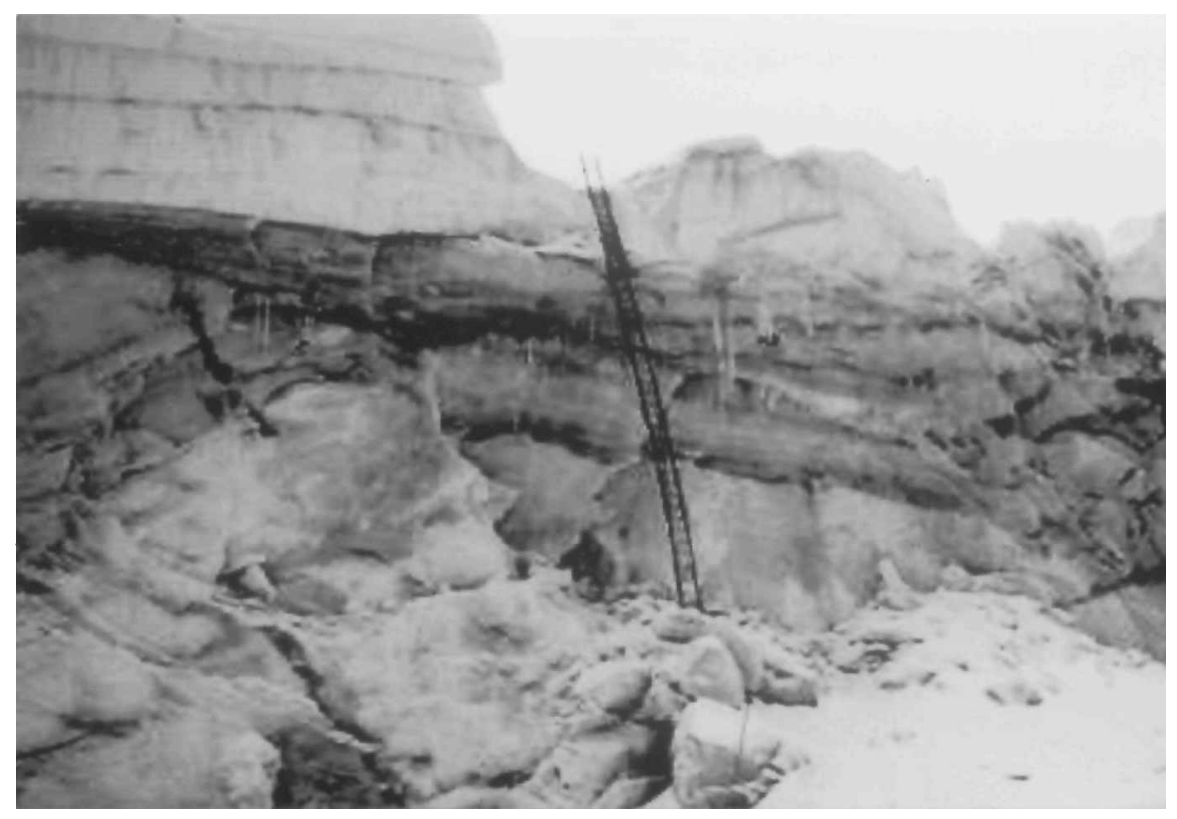

FIG. 6. The lower face of the Khumbu Icefall, with aluminum ladders lashed together to allow for the ascent. (Courtesy of Rick Satava, Jr., Base Camp Manager, Boulder, Colorado.) 


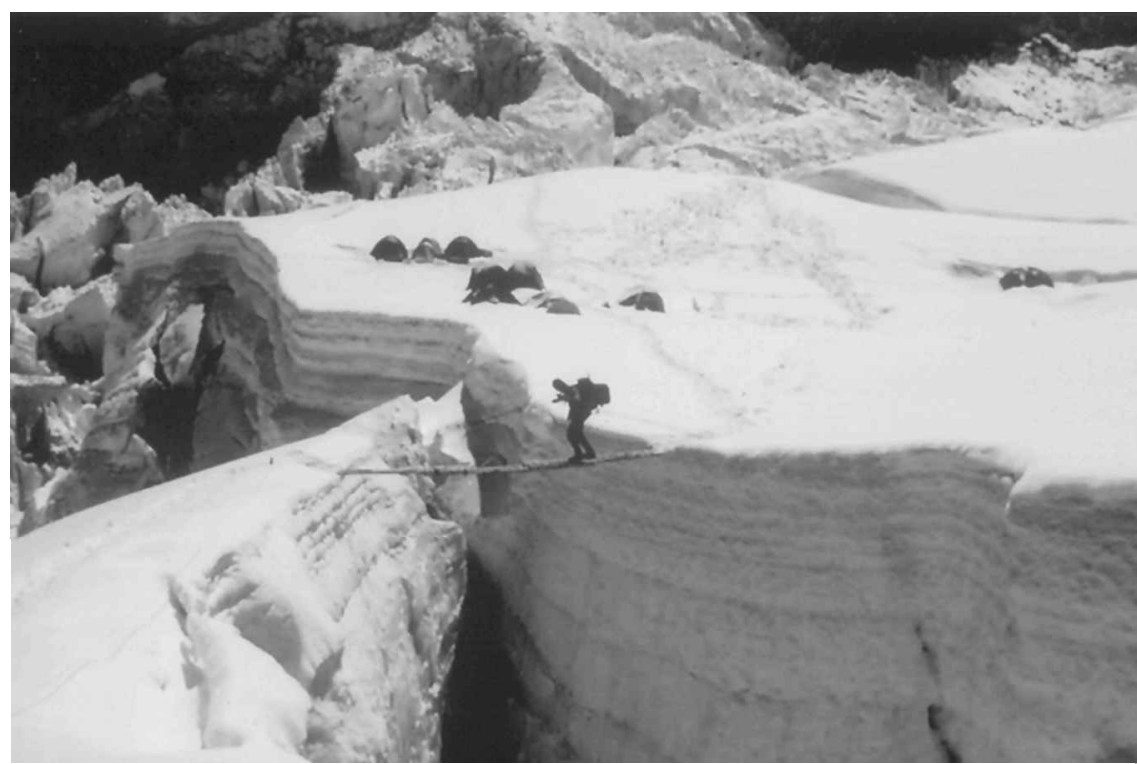

FIG. 7. Crossing a deep crevasse on the Khumbu Ice-fall, using ladders roped together. (Courtesy of James Williams, Climbing Team Leader, Jackson, Wyoming.)

\section{RESULTS}

Figure 4 illustrates the home screen of the laptop computer that was the interface for viewing the data from the climbers. On the left-hand side is a scale map of the terrain between EBC and Camp 1, demonstrating the trail and location of an individual climber. The large concentration of data points in the upper left is at Camp 1, where the climbers established camp and remained overnight. One small excursion slightly beyond Camp 1 for purposes of photographic documentation can be noted heading diagonally to the upper left corner. In addition, there is a large skew of one climber position due to malfunction (most likely a miscalculation of GPS signal capture). The inset in the lower left corner is a reference graphic representation of the vertical ascent usually taken to the summit, with the numbers representing the locations of the four camps. On the right side of the screen are the "thumbnail" graphics of the continuous vital-signs summaries of the three climbers (two being active at the time of the screen capture) along with their latest updated values. Clicking on any of the climber boxes takes you to the detailed individual vital-signs screen (Fig. 5), which provides specific overall information as well as the chronological, high-fidelity presentation of data points acquired in real time and plotted every 5 minutes. To provide as robust data acquisition as possible, standard strategies were employed such as continuous resampling and graceful degradation. Each sensor was polled 4 times per minute, and once the datum point for the 5-minute interval was acquired, it was sent to the hub where it was stored with all the other sensor data. At 5-minute intervals, the hub data "cache" was read and transmitted to the base station, as well as archived for later analysis. Missed data were stored as either a " 0 " value or a default datum point. The data were monitored in real time and stored on both the receiving computer and the wearable data logger. Whenever there was loss of transmission, the data logger would continue to store the data and then retransmit the entire data from the last successful transmission.

\section{Reliability of data acquisition and transmission}

The GPS location functioned well for two of the three climbers. Climber 1 initially had an excellent functioning system. After a short time, however, the system became erratic in location acquisition and on the reascent totally 
Table 1. Typical Dataset from Climber 3 While Climbing in the Khumbu Icefall

\begin{tabular}{|c|c|c|c|c|c|c|c|}
\hline Data ID \# & Time (GMT) & Latitude & Longitude & Heart rate & Activity & Skin temp & Core temp \\
\hline 3378 & $12: 17: 46$ & 28.007 & 86.86044 & 92 & 30 & 11.02 & 38.23 \\
\hline 3379 & $23: 22: 43$ & 28.00663 & 86.86144 & 96 & 19 & 16.24 & 37.85 \\
\hline 3380 & $23: 22: 43$ & 28.00658 & 86.86138 & 96 & 16 & 22.11 & 37.36 \\
\hline 3381 & $23: 35: 47$ & 28.00725 & 86.86089 & 84 & 27 & 24.11 & 37.12 \\
\hline 3382 & $23: 40: 35$ & 28.00704 & 86.86126 & 84 & 48 & 25.16 & 37.27 \\
\hline 3383 & $23: 45: 47$ & 28.00716 & 86.85998 & 148 & 44 & 25.85 & 37.95 \\
\hline 3384 & 23:50:47 & 28.00625 & 86.86282 & 128 & 36 & 26.55 & 37.67 \\
\hline 3385 & 0:00:01 & -37.3914 & 122.0376 & 128 & 37 & 27.68 & 37.61 \\
\hline 3386 & 0:00:01 & 26.63363 & 29.78837 & 124 & 48 & 29.7 & 37.53 \\
\hline 3387 & $0: 05: 47$ & 28.00522 & 86.86506 & 100 & 18 & 31.19 & 37.45 \\
\hline 3388 & $0: 10: 47$ & 28.0039 & 86.86366 & 160 & 44 & 31.71 & 37.36 \\
\hline 3389 & $0: 15: 47$ & 28.00399 & 86.86554 & 168 & 49 & 32.36 & 37.53 \\
\hline 3390 & $0: 20: 47$ & 28.00293 & 86.86737 & 172 & 38 & 32.86 & 37.8 \\
\hline 3391 & $0: 25: 47$ & 28.00216 & 86.86763 & 172 & 30 & 33.05 & 37.95 \\
\hline 3392 & $0: 30: 47$ & 28.00199 & 86.86862 & 176 & 37 & 33.18 & 38.13 \\
\hline 3393 & $0: 35: 47$ & 28.00154 & 86.86896 & 172 & 35 & 33.25 & 38.21 \\
\hline 3394 & $0: 40: 47$ & 28.00136 & 86.86934 & 180 & 38 & 33.34 & 38.23 \\
\hline 3395 & $0: 45: 47$ & 28.00012 & 86.87052 & 176 & 32 & 33.16 & 38.26 \\
\hline 3396 & $0: 50: 47$ & 27.9993 & 86.8708 & 172 & 26 & 33.16 & 38.23 \\
\hline 3397 & $0: 55: 47$ & 27.99964 & 86.87203 & 172 & 31 & 33.13 & 38.26 \\
\hline 3398 & 1:00:41 & 27.99952 & 86.87135 & 172 & 26 & 33.01 & 38.26 \\
\hline 3399 & 1:05:46 & 27.99786 & 86.87095 & 172 & 31 & 32.94 & 38.15 \\
\hline 3400 & $1: 10: 46$ & 27.99752 & 86.87148 & 168 & 26 & 32.96 & 38.08 \\
\hline 3401 & $1: 15: 47$ & 27.99665 & 86.87207 & 156 & 37 & 32.74 & 38.02 \\
\hline 3402 & $1: 20: 47$ & 27.99625 & 86.87303 & 164 & 29 & 32.64 & 38.06 \\
\hline 3403 & $1: 25: 47$ & 27.99513 & 86.87352 & 168 & 33 & 32.46 & 38.26 \\
\hline 3404 & 1:30:45 & 27.99513 & 86.87316 & 168 & 35 & 32.28 & 38.04 \\
\hline 3405 & $1: 35: 44$ & 27.9941 & 86.87302 & 152 & 32 & 31.93 & 38.08 \\
\hline 3406 & $1: 40: 37$ & 27.99466 & 86.8732 & 168 & 27 & 31.79 & 37.93 \\
\hline 3407 & $1: 45: 47$ & 27.99372 & 86.87372 & 136 & 21 & 31.75 & 37.93 \\
\hline 3408 & $1: 50: 47$ & 27.99415 & 86.87329 & 152 & 35 & 31.83 & 38.02 \\
\hline 3409 & 1:55:47 & 27.99523 & 86.87547 & 156 & 39 & 31.94 & 37.95 \\
\hline
\end{tabular}

Time is Greenwich Mean Time (GMT). Heart rate is in beats per minute. Activity is in motions per minute. Skin and core temperatures are in degrees Celsius.

failed. The two other systems continued to function perfectly well at the same time and place. The reliability of acquiring location on the two functioning GPS systems for continuous monitoring was $100 \%$. In the third system, data acquisition was initially $75 \%$ (9 of 12 accurate locations) in the first hour; however, it rapidly degraded to $36 \%$ acquisition (18 of 50 data points) in the next 4 hours, then quit altogether. The vital-signs data had a loss of transmission rate from $3 \%$ to $12 \%$, with the exception of one climber who must have had an improper affixing of the leads for the heart rate and activity monitor because of erratic loss of signal with only $56 \%$ data acquisition during the later descent portion of the trek. However, no vital-signs signals were lost for more than 35 minutes or seven serial recordings. In all proper functioning monitors, no signal was lost for more than four consecutive readings or
20 minutes. This occurred on only one occasion.

\section{Vital signs data}

The vital-signs monitors functioning from $95 \%-100 \%$ of the time, with the exception of one climber whose heart rate monitor functioned $78 \%$ of the time. The heart rate varied from 76 beats per minute at rest to 176 at strenuous exercise. The climbers had different baseline heart rates before the ascent, in the 100 to 120 beats per minute range, which corresponded to their resting heart rate at the EBC after acclimatization. The Sherpa had a lower baseline heart rate of $86-100$ beats per minute. All climbers experienced an increase in heart rate to the 150 beats per minute range, often (but not always) correlating to significant increase in the actigraph which indicated signif- 
icant exercise. The skin temperature sensor functioned extremely well, even during a time when there was loss of signal from the temperature pill (see below). The maximum variability of skin temperature of $22.1^{\circ} \mathrm{C}$ to $34.3^{\circ} \mathrm{C}$ (usually $5-10^{\circ} \mathrm{C}$ over the duration of a climb, which was monitored over 6-9 hours) was much greater than the core temperature of $36.7^{\circ} \mathrm{C}$ to $39.6^{\circ} \mathrm{C}$, but it was usually within $4-7^{\circ} \mathrm{C}$ of core body temperature. The one exception was when one climber had improper position or an open overcoat at the beginning of the reascent, when there was abnormally low $\left(11-21^{\circ} \mathrm{C}\right)$ readings. This was corrected, and it functioned well throughout the remainder of the climb. There was no direct proportionality between the skin and core body temperature, and there were a few times when skin temperature changed in the opposite direction from core temperature. The core temperature pill was extremely accurate and fluctuated only $1-3^{\circ} \mathrm{C}$ over the duration of the climb. There was one climber in whom the pill suddenly stopped functioning in the middle of giving normal stable readings. It is not known if the pill simply stopped working or was possibly excreted. An interesting phenomenon with the temperature pill is that it is possible to tell when the climber is taking a drink of liquid (either hot or cold) because there is a sudden change (usually $1-3^{\circ} \mathrm{C}$ ) in pill temperature. Intermeasurement change was not greater than $\pm 0.5^{\circ} \mathrm{C}$ per 5-minute interval, even with strenuous activity. The correlation between heart rate, activity level, skin temperature, and core temperature was not consistent, though there were numerous intervals of 10-20 minutes when a sudden dramatic increase in heart rate was accompanied by a rise in actigraph level, skin temperature, and even a gradual and persistent increase in core body temperature. Unfortunately, the strenuous climb did not permit an event recorder to indicate what any individual climber was doing during the sudden change in vital signs.

\section{DISCUSSION}

In April 1999, a workshop of Home Care Technologies for the 21st Century sponsored by the National Science Foundation and the Cen- ter for Devices and Radiologic Health of the Food and Drug Administration reported that it is "anticipated that health care will migrate to a more proactive, preventative model rather than reactive, episodic model utilized today [with]: Intelligent wearable sensors, trend analysis tools, predictive algorithms. ${ }^{5}$ There seems to be a consensus that wearable, wireless transmission of health data is commonplace. However, there are few reports in this area.

The ambulatory monitoring of EKG signals can be traced to the Holter monitor. However, this wearable system stored the data in a beltworn recorder for analysis 24 hours later. The concept of a wearable computer that could receive and transmit information in real time was conceived by Steve Mann of the Massachusetts Institute of Technology Media Lab in the late 1970s, and it developed into what is now recognized as "wearable computing."6 This brought to the forefront the possibility of continuously monitoring and transmitting medical information on patients. In 1986 Shichiri et al. ${ }^{7}$ attempted continuous monitoring of glucose concentration in five diabetic patients with a needle-type glucose sensor that transmitted the signals. This prompted the development of a wearable glucose monitoring systems by Fabietti et al. ${ }^{8}$ and Pfeiffer, ${ }^{9}$ but difficulties persisted with signal to noise and reliability of sensors. In 1991, Mittal et al. ${ }^{4}$ reported the first use of an ingested temperature pill that telemetered the signals to a belt-worn recorder and described its characteristics. This pill acquired core body temperature with an accuracy of $0.04^{\circ} \mathrm{C}$ over a range of $30-50^{\circ} \mathrm{C}$. The ingested temperature pill has had a number of applications within the research community but has not been routinely employed in clinical practice. Novel wearable approaches to monitor blood lactate by Meyerhoff et al., ${ }^{10}$ ethanol by Swift et al., ${ }^{11}$ and clotting of an arteriovenous fistula for dialysis by Shinzato et al. ${ }^{12}$ once again demonstrated the feasibility of such systems. Due to several factors, however, including accuracy, power requirement, signal acquisition, and poor ergonomics, these systems have not achieved common clinical use. In 1997, Richey et al. ${ }^{13}$ compared ambulatory monitored blood pressure measurements to traditional techniques in 216 patients. Ninetyone percent of the recordings were successfully 
acquired, and $81 \%$ of the measurements were interpretable. However, these systems are not user friendly. They are cumbersome and larger than desired for comfortable use. Their deployment has only been in feasibility studies or unique circumstances. Surprisingly, there have been no reports of VSM with remote transmission of real-time signals using wireless wearable systems. An interesting test was conducted on a commercial aircraft in July 1997 by Gandsas and Montgomery ${ }^{14}$ in which a commercial VSM system (Propaq 106, Protocol Systems Inc., Beaverton, OR) connected an EKG on a volunteer to a cellular phone on an aircraft through a computer interface (with a 4,800 baud rate). Vital signs were recorded using 3lead EKG, and blood pressure, pulse, respiration, and oxygen saturation were monitored over the Internet "without any corruption of data with an average delay time of $15 \mathrm{sec}$." The signals were transmitted during a flight at 35,000 feet between Los Angeles and Chicago to receiving stations at Saddle Back Memorial Hospital (Laguna Hills, CA) and Santojanni Hospital (Buenos Aires, Argentina). This clearly demonstrated the feasibility (though rather cumbersome) of transmitting vital signs from remote sites without terrestrial connectivity. However, the signal acquisition systems were not wearable. There are no subsequent reports regarding further research of this type of system or whether the airline industry is using it.

There are no documented reports of continuous real-time monitoring of vital signs on an ambulatory person in truly remote or hazardous conditions. Therefore, one of the goals of this expedition was to assess a physiologic cipher and to validate the technical feasibility of real-time monitoring of the vital signs and geolocation position of individual climbers in a real-world, extreme environment. The system was robust, fault tolerant (resampling when a GPS signal was not acquired or when a vital sign was not detected), and easily monitored through the graphical interface. The obvious physical stress of hypoxia and strenuous activity were seen in the overall elevated baseline heart rate. As the climbers ascended, however, there were no specific event markers to correlate level of exercise or effort with heart rate. The skin and core body temperatures showed different variations. As expected, skin temperature had a wider variation than did core temperature, and, at the outside air temperature and level of activity, the core body temperature was easily maintained and the skin temperature was only modestly affected (usually $3-9{ }^{\circ} \mathrm{C}$ less than core temperature).

There were several episodes of lack of signal acquisition. However, the frequent sampling (every 15 seconds) provided adequate compensation when signal acquisition was momentarily lost. The values obtained compared appropriately with the values derived from the person a few days before the climb at EBC. Data transmission from the individual to EBC and retransmission in real time to Yale University validated the concept of remote (or perhaps even global) monitoring of individuals. Although there was no methodology to verify that the GPS location of the climbers correlated with their exact location, their position at Camp 1 was accurate. The weather was clear during the trek up to Camp 1 and slightly overcast on the return down. The effects of severe weather, such as a snowstorm, on the quality of transmission were not determined.

This expedition exemplifies the extreme conditions for operating a VSM system over which there is nearly no control. There are numerous applications of VSM, such as prehospital and in-hospital settings, nursing homes, or even astronauts in space, but more so when the conditions are not nearly so extreme and the particular environment allows for control of factors that would otherwise degrade the acquisition of signals. Most hospitals can secure an excellent communications system for monitoring vital signs by a wireless system. As more parameters are added to such a system through the implementation of noninvasive sensors, the physiologic cipher will become less of a mystery and more of a quality control asset.

\section{ACKNOWLEDGMENTS}

Support for this research was provided through grants from Olympus America, Inc., Yale University-NASA Commercial Space Center for Medical Informatics and Technology 
Applications (NASA NCC5-191), and the Saint Charles Hospital in Port Jefferson, New York.

A review of this project also appeared in the Yale Journal of Biology and Medicine (1999; 72:19-27), and any similarities are unintentional. The authors received permission to publish the current manuscript.

\section{REFERENCES}

1. Krakauer J. Into thin air (illustrated ed.). New York: Villiard Books, 1998:322-337.

2. Coburn B, Beshears D. Everest: Mountain without mercy. Willard, OH: National Geographic Society Press, 1997:76-87.

3. Satava, RM. Virtual reality and telepresence for military medicine. Comput Biol Med 1995;25(2):229-236.

4. Mittal BB, Sathiaseelan V, Rademaker AW, Pierce MC, Johnson PM, Brand WN. Evaluation of an ingestible telemetric temperature sensor for deep hyperthermia applications. Int J Radiat Oncol Biol Phys 1991;21(5):1353-1361.

5. Dighe A, Warren S. Smart health care systems and the home of the future. In: Winters J, Herman W, eds. Workshop on home care technologies for the 21st century. http:// www.hctr.be.cua.edu/hctworkshop/HCTr_F.htm (Originally accessed June 23, 2000.)

6. Mann S. An historical account of the "WearComp" and "WearCam" inventions developed for applications in "Personal Imaging." In: Bass L, Pentland A, eds. The first international symposium on wearable computers (Oct 1997). Los Alamitos, CA: IEEE Computer Society, 1997:66-73.

7. Shichiri M, Asakawa N, Yamasaki Y, Kawamori R, Abe $\mathrm{H}$. Telemetry glucose monitoring device with needle-type glucose sensor: A useful tool for blood glucose monitoring in diabetic individuals. Diabetes Care 1986;9(3):298-301.
8. Fabietti PG, Massi Benedetti M, Bronzo F, Reboldi GP, Sarti E, Brunetti P. Wearable system for acquisition, processing and storage of the signal from amperometric glucose sensors. Int J Artif Organs 1991;14(3): 175-178.

9. Pfeiffer EF, Meyerhoff C, Bischof F, Keck FS, Kerner $\mathrm{W}$. On line continuous monitoring of subcutaneous tissue glucose is feasible by combining portable glucosensor with microdialysis. Horm Metab Res 1993;25 (2):121-124.

10. Meyerhoff C, Bischof F, Mennel FJ, Sternberg F, Bican J, Pfeiffer EF. On line continuous monitoring of blood lactate in men by a wearable device based upon an enzymatic amperometric lactate sensor. Biosens Bioelectron 1993;8(9-10):409-414.

11. Swift RM, Martin CS, Swette L, LaConti A, Kackley N. Studies on a wearable, electronic, transdermal alcohol sensor. Alcohol Clin Exp Res 1992;16(4):721-725.

12. Shinzato T, Nakai S, Takai I, Kato T, Inoue I, Maeda $\mathrm{K}$. A new wearable system for continuous monitoring of arteriovenous fistulae. ASAIO J 1993;39(2):137140.

13. Richey PA, Jones CL, Harshfield GA, Somes GW, Johnson KC, Bailey JE, Soberman JE. The AM5600 ambulatory blood pressure recording system. Blood Press Monit 1997;2(4):193-195.

14. Gandsas A, Montgomery K, McKenas D, Altrudi R, Silva Y. In-flight continuous vital signs telemetry via the Internet. Space Environ Med 2000;71(1):68-71.

Address reprint requests to: Richard M. Satava, M.D., F.A.C.S. Yale University School of Medicine 40 Temple Street, Suite 3-A New Haven, CT 06510

E-mail: richard.satava@yale.edu 